\title{
Impacto del fomento de una cultura de emprendimiento en los aprendices del programa de tecnología en gestión administrativa en el centro de la industria, la empresa y los servicios del Sena (Huila)
}

\author{
Carlos Enrique Parra Rodríguez \\ SENA, Neiva \\ cparra@sena.edu.co \\ Hernando Gil Tovar \\ Universidad Surcolombiana, Colombia \\ hergil@usco.edu.co \\ Maber Rengifo Gonzales \\ mabery154@gmail.com
}

\section{Resumen}

Este artículo tiene como propósito presentar un análisis sobre un espacio para la medición del impacto de las actividades de formación en emprendimiento y su interrelación entre la investigación, la creatividad y la innovación, el cual permitirá el fortalecimiento del emprendimiento en los aprendices de la Centro de la Industria, la empresa y los Servicios (Sena Huila) sede Neiva, con las posibilidades de asociarlos a planes o a Investigaciones de negocios futuras, que en esencia ratificaría el carácter de formación a nuestra Institución en Neiva.

Este proyecto busca conocer los esfuerzos realizados en la formación para el emprendimiento y su impacto en la cultura emprendedora, insumo posterior para la búsqueda de la participación activa de los aprendices Tecnólogos del programa de Tecnología en Gestión Administrativa de la Centro de la Industria, la empresa y los Servicios (Sena Huila) en la sede de Neiva, con la colaboración de gremios, empresario y el gobierno, donde se puedan impulsar procesos académicos y empresariales.

Palabras claves: Iniciativa empresarial, creación de empresas, estructuras curriculares, segmento curricular.

\section{Impact of the promotion of a culture of entrepreneurship in the apprentices of} the program of technology in administrative management in the center of the industry, the company and the services of the SENA (Huila)

\section{Summary}

This article aims to present an analysis of a space for measuring the impact of training in entrepreneurship and its interface between research, creativity and innovation, which will allow the strengthening of entrepreneurship in students of the Center Industry, business and Services (Seine Huila) based Neiva, the possibilities of associating Research plans or future business, which essentially ratify the character of our institution training in Neiva. 
This project seeks to understand the efforts in training for entrepreneurship and its impact on entrepreneurial culture, back to seeking the active participation of students in the program Technologists Technology in Administrative Management Industry Center input, the company and Services (Seine Huila) at the headquarters of Neiva, in collaboration with unions, business and government, where they can boost academic and business processes.

Keywords: Entrepeneurial initiative, Business Foundation, Curricular Structures, Curricular Segments.

\section{Introducción}

El emprendimiento es clave para el desarrollo económico de un país, departamento, municipio o región, es la sangre nueva que se inyecta a la economía todos los años. Crea empleos, da oportunidades de desarrollo social y fomenta la producción dentro de la economía.

El emprendimiento es una forma de pensar, razonar y actuar centrada en las oportunidades, planteada con visión global y llevada a cabo mediante un liderazgo equilibrado y la gestión de un riesgo calculado, su resultado es la creación de valor que beneficia a la empresa, la economía y la sociedad. (Ley 1014 de 2006, art. 1ํㅡ).

Es por esto que el fomento al emprendimiento es un objetivo más que relevante para la política de desarrollo empresarial.

Como todo en el mundo de las políticas públicas es necesario hacer un buen diseño de éstas de manera que puedan cumplir con los objetivos planteados y sean eficientes en el logro de éstos. Asímismo la evaluación de éstas cobra una gran importancia al ser la herramienta capaz de iluminarnos sobre la calidad de nuestra política como motor de los cambios que busca generar.

Vivimos en un mundo donde las riquezas son escasas y las necesidades son múltiples, se debe tomar en cuenta también la necesidad de utilizar de forma correcta y eficiente los mismos con los que se cuenta por lo que las herramientas que se utilicen, deben cumplir también las características que deseamos encontrar en nuestras políticas públicas.
En los establecimientos educativos, el emprendimiento, el emprendimiento, desde un enfoque de desarrollo humano integral es entendido como una forma de pensar, sentir y actuar para la creación de valor, lo cual permite a la comunidad educativa proponer espacios y escenarios de formación para:

- Construir conocimientos y desarrollar hábitos, actitudes y valores necesarios para generar acciones orientadas al mejoramiento personal y a la transformación del entorno y de la sociedad;

- Dar soluciones a las necesidades humanas presentes en la comunidad, con un sentido de ética y responsabilidad social y una perspectiva de desarrollo sostenible;

- Promover la cooperación y el trabajo en equipo en todos los miembros de la comunidad;

- Fortalecer en los aprendices la capacidad de conseguir y conservar un empleo, acceder a diferentes alternativas laborales y a posibilidades de autoempleo;

- Consolidar procesos de articulación del establecimiento educativo con el

sector productivo, la educación superior y la educación para el trabajo.

Por lo tanto es necesario recordar el concepto de emprendimiento; acometer y comenzar una obra, un negocio, un empeño, especialmente si encierran dificultad o peligro. (Real Academia Española, 2001). Por consiguiente se entiende por emprendimiento como un acto que involucran riesgos y que requiere de algo de experiencia, con incentivos fuertes que vengan desde un plano interno del individuo como de un plano externo en el entorno que lo rodea ya 
que siempre estará condicionado por las oportunidades y alternativas que éste encuentre. Dado el supuesto de que los individuos son racionales, jamás emprenderán si encuentran una alternativa que perciban de forma más atractiva.

Dentro de la literatura que hay respecto al tema, encontramos el modelo de Shapero (1975) quien plantea que existen tres factores fundamentales para el emprendimiento:

En primer lugar, deseabilidad percibida: Qué tan atractiva es la idea en qué consiste el emprendimiento para el individuo. En segundo lugar, viabilidad detectada: Qué tan viable es el emprendimiento dentro de las posibilidades y condiciones con las que cuenta el individuo. Por último, propensión a actuar: La capacidad de decidirse por realizar el emprendimiento. Tiene que ver con la rapidez en la toma de decisiones y el grado de aversión al riesgo. (p. 83)

En el año 1803 el economista francés Jean Baptista Say (citado por Varela, 2001) dio a conocer la primera diferenciación entre los términos empresario y capitalista afirmando que

El empresario es el agente que reúne y combina los medios de producción (recursos naturales, recursos humanos y recursos financieros) para constituir un ente productivo, y encuentra en el valor recibido de los productos, la recuperación del capital que el emplea, de los gastos en que incurre y de la utilidad que busca. Es el punto de apoyo (pibot) sobre el cual gira todo el negocio. Para ser exitoso debe tener juicio, perseverancia y un conocimiento tan bueno del mundo como del negocio. Debe poseer el arte de la superintendencia y de la administración. (p. 90)

De esta afirmación se puede concluir que el empresario es el principal agente y el actor fundamental de las actividades de producción, capaz de vencer las dificultades presentadas por el entorno diariamente. Esto también se puede evidenciar según las afirmaciones realizadas por Arthur Cole en 1959 (citado por Varela, 2001) ser empresario es la actividad personal de un individuo o grupo de individuos que toman la iniciativa, mantienen y crecen una unidad de negocio orientada a la producción y distribución de bienes y servicios.

El emprendimiento se ha establecido en los últimos años como la base del desarrollo y crecimiento de un país debido a que trae consigo nuevas ideas y más empresas productivas que le aportan una dinámica a la sociedad. Aun así, es de vital importancia entender que el emprendimiento debe ir obligatoriamente ligado a la educación y debe ser desarrollado en todos los ámbitos, tanto en el entorno como en el personal. Lo que se busca en últimas es lograr, después de un largo proceso, que el tema de emprendimiento sea considerado como un elemento estratégico en el mejoramiento continuo de cada país y de esta forma aprovechar cada uno de los beneficios que trae consigo el desarrollo.

\subsection{Problemática Objeto de estudio}

En la reciente Conferencia Mundial de la Educación Superior realizada en Paris (2009) contempla en su declaración final la responsabilidad social de la Universidad como una de las estrategias que marcaran rumbo de las instituciones de educación superior en las próximas décadas. Se precisa, que la educación superior como un bien público es responsabilidad de todos los miembros de la sociedad, especialmente de los gobiernos. Encarando la complejidad de los desafíos globales presentes y futuros, la educación superior tiene la responsabilidad social de incrementar nuestro entendimiento en distintas dimensiones como son lo social, económico, científico y cultural; así como nuestra capacidad de respuesta frente a ellas.

El papel del emprendimiento debe ser clave para lograr la mejoría en las regiones huilenses y este proceso está en manos de las instituciones públicas a nivel educativo como las universidades privadas como públicas (Centro de la Industria, la empresa y los Servicios (Sena Huila), Alcaldías Municipales, Departamento del Huila, Sena, entre otras) quienes tienen bajo su responsabilidad la preparación de alumnos capaces de enfrentarse y competir con un mercado laboral demasiado 
competitivo, logrando utilizar las herramientas dadas para aportar al desarrollo económico y social de un país.

En el caso particular de la Tecnología en Gestión Administrativa en la Centro de la Industria, la empresa y los Servicios (Sena Huila) (Sede Neiva), se ha esforzado por inculcar el tema de emprendimiento en cada uno de sus aprendices, lo cual se ve reflejado en el aprendizaje por proyectos. Aun así no existe un sistema en donde se encuentren consolidados los resultados de la información anterior por lo cual no se pueden analizar con verdadera certeza los efectos obtenidos en los aprendices para así reforzar y mejorar las falencias y al mismo tiempo resaltar lo que se está haciendo de la manera correcta. Lo que se busca entonces al realizar este trabajo, es analizar con talleres dirigidos el grado con respecto al tema de emprendimiento de los aprendices de Tecnología en Gestión Administrativa para verificar la importancia que estos tienen en su vida profesional y así poder establecer el impacto que se ha tenido en los aprendices. Además se hace necesario realizar un estudio en el cual se evidencien los casos exitosos y se expongan los factores que han llevado a estos aprendices a alcanzar dicho éxito.

¿Cuál es el impacto de las actividades de fomento de la cultura de emprendimiento en los aprendices del programa de Tecnología en Gestión Administrativa en la Centro de la Industria, la empresa y los Servicios (Sena Huila) en la sede de Neiva (Huila) (2015)?.

\section{Objetivos}

Para el desarrollo de esta investigación persigue los siguientes objetivos:

\section{Objetivo General}

- Determinar el impacto en la formación de emprendimiento en los estudiantes del programa de administración de empresas en la universidad Surcolombiana en la sede de Pitalito (Huila) (2009 - 2012).

\section{Objetivos Específicos}

- Realizar un inventario de los proyectos de emprendimiento realizados en la sede de Pitalito con los estudiantes de Administración de Empresas de la Universidad Surcolombiana durante los años (2009 - 2012).

- Observar los Impactos de generación de empleo o incremento en el volumen de las ventas generados por las nuevas ideas de negocios de los estudiantes de Administración de Empresas durante los años (2009 - 2012).

- Identificar estructuras comerciales de los estudiantes activos que permita desarrollar un entorno económico - social y que se pueda utilizar como criterios de progreso en la región.

- Sensibilizar y capacitar a los estudiantes $y$ docentes de la comunidad universitaria en la sede de Pitalito sobre la importancia de participar con propuestas creativas, tales como ferias, a través de la divulgación y promoción de dichos eventos.

- Realizar Iniciativas empresariales documentales con elementos

innovadores y/o tecnológicos para puedan obtener apoyo en su realización.

- Generar estrategias y acciones para desarrollar las actitudes emprendedoras en los estudiantes del programa de Administración de Empresas de la Universidad Surcolombiana en la Sede de Pitalito, con el fin de fomentar la cultura del emprendimiento.

- Integrar y fortalecer las competencias de trabajo en equipo, mediante la implementación y realización de una pequeña muestra empresarial y rueda de inversiones.

\section{Metodología}

En cumplimiento de los objetivos propuestos y la naturaleza del estudio se desarrolló un enfoque basado en la complementariedad, a partir de integración de acciones relacionadas con la revisión teórica y conceptual, el análisis de documentos oficiales e institucionales, la caracterización del objeto de estudio teniendo como referencia la consulta a los actores que participaron. 
Para poder proponer el impacto como el que se detalla en el objetivo del presente estudio, es necesario entender primero a qué se refiere como Evaluación de Impacto y sus diferentes niveles con el fin de aplicar correctamente la metodología que se utilizará durante el desarrollo de éste.

Para este análisis se ha utilizado el estudio secundario como tipo de investigación (Yin, 1994). Esta metodología es imprescindible en las primeras etapas de la comprensión de un fenómeno (Cepeda, 2003). Los estudios basados en casos son habituales en investigaciones exploratorias, sobre todo, en entornos y organizaciones complejas y, generalmente, son empleados por investigadores de Tecnología en Gestión Administrativa (ídem). El análisis exploratorio se centró en literatura de la tipología dada por investigadores a partir de resultados obtenidos de sus investigaciones y en el análisis empírico resultado de un estudio de caso, sobre emprendedores amparado en unas incubadoras de empresas y una muestra de emprendedores no cobijados bajo modelos de incubación.

\subsection{Elementos cuantitativos y cualitativos}

La evaluación de resultados ve elementos cualitativos y cuantitativos del programa. Enel caso de los programas de emprendimiento, los aspectos cuantitativos se verán reflejados en indicadores como: cantidad de emprendimientos por curso intervenido y cantidad de emprendimientos que sobreviven después del primer año entre otras variables relevantes. Por otra parte, en los aspectos cualitativos veremos reflejados indicadores como: grado de aceptación por los alumnos de las herramientas entregadas y cambios en la percepción del emprendimiento de los alumnos entre otras variables relevantes.

\subsection{Relación causa- efecto}

Dentro de la metodología es importante establecer una hipótesis causal que vea la causalidad que tiene el programa en sí dentro del cumplimiento de los objetivos establecidos, la relación causa-efecto se convierte en un elemento esencial dentro de la evaluación de impacto. Evaluando esta causalidad a lo largo de la implementación del programa, es posible saber si ciertos elementos o componentes del programa son relevantes para el logro de los objetivos planteados y así ir perfeccionándolo sobre la marcha. De esta forma se podrá tener una mirada objetiva en torno al logro de las metas propuestas y se evitará un "exceso de equipaje" en el desarrollo del proyecto.

\subsection{Horizontes de tiempo}

Dada la multidimensionalidad de los programas y la diversa naturaleza de sus componentes, es esperable que los efectos del tratamiento se hagan visibles en diferentes horizontes de tiempo por lo que debe ser capaz de separar los efectos de corto, mediano y largo plazo. Una vez que se identifiquen y separen, se debe ser oportuno en la evaluación de estos para poder tener una idea general sobre los efectos que está produciendo el programa y la incidencia y relevancia de estos para el logro de los objetivos planteados.

\subsection{Influencia de factores externos}

Los resultados a nivel de producto y los resultados intermedios obedecen a consecuencias directas de los productos y servicios que genera y entrega el programa, por lo que están vinculados netamente a factores internos del programa. Sin embargo, a nivel de resultados finales se debe ser capaz de identificar a los factores externos que puedan influir en el objetivo del programa y los efectos que estos factores tengan en el grupo objetivo. Un ejemplo de factor externo relevante para el caso del emprendimiento son los ciclos económicos ya que pueden tener una incidencia directa en el éxito o fracaso de un emprendimiento. Es importante separar los factores externos que se pueden controlar de los factores externos que no se pueden controlar. Un factor externo controlable sería por ejemplo un programa de otro ejecutor con objetivos y productos similares que eventualmente puedan influir en el comportamiento de nuestro grupo objetivo.

Existen técnicas dentro de la evaluación de impacto que permiten aislar estos factores del 
análisis de los resultados. Estas técnicas serán analizadas más adelante.

\section{Resultados}

La medición y evaluación de resultados a nivel de producto, dada la naturaleza de éste resultado, debe hacerse de forma cuantitativa. De modo que corresponde a cantidades o porcentaje de entregas de los productos o servicios que generan los componentes del programa. Hay que contar con una definición amplia de producto que permita una evaluación más completa en que se puedan agregar características del proceso en sí como la oportunidad en que se entregan los

A nivel de resultados intermedios, la evaluación apunta a identificar cambios en el comportamiento de los individuos tratados que se produzcan a partir del producto o servicio entregado. Analizan la adquisición de ciertas competencias, herramientas y habilidades que contribuirán finalmente a los objetivos y metas trazadas que se evaluarán después a nivel de resultados finales. En esta evaluación interesa saber si los conocimientos y herramientas entregadas están siendo usados de manera correcta, es una forma de ver la efectividad del aprendizaje en los individuos tratados. A diferencia de la evaluación de producto, la información relevante no se encuentra en el programa en sí sino que debe obtenerse a través de herramientas adicionales que recojan la información necesaria para la construcción de los indicadores, por ejemplo una encuesta.

Para el caso de la medición de los resultados finales, lo primordial es identificar los efectos que tiene el tratamiento a mediano y largo plazo ya que lo que interesa ver a este nivel son los beneficios perdurables en el tiempo con los que se quedará la población objetivo. En el caso del emprendimiento se quiere saber el impacto económico que tendrá este programa en el largo plazo para la población objetivo y eso es lo primordial debido a que si se entregan productos y servicios y estos productos y servicios generan cambios conductuales en el grupo objetivo, no sirven demasiado si es que no contribuyen al fin o al propósito del programa, lo que debe estar establecido en su diseño. El fin de las políticas públicas y programas debe ser siempre generar un cambio positivo y una mejora en alguna característica de la población que ha sido identificada anteriormente.

La construcción de resultados por niveles, desde los inmediatos (producto) e intermedios debe llevar a resultados finales consecuentes con el fin establecido ya que de lo contrario se considerará que la política pública o el programa han fracasado en su capacidad como herramienta de transformación social.

Para medir esto, lo primero es generar los indicadores con los que se podrá ver si el programa ha tenido el impacto esperado. El impacto del programa corresponderá a la diferencia en la variable del resultado $(\mathrm{Y})$ entre el individuo con tratamiento y la variable resultado para el mismo individuo sin tratamiento, es decir, captura el efecto neto del programa.

Si se toma como la situación final del individuo " $\mathrm{i}$ " con tratamiento y se toma como el resultado final del individuo " $\mathrm{i}$ " sin el tratamiento, se obtiene que el efecto neto del tratamiento sobre el individuo " $\mathrm{i}$ ", ai es igual a:

$$
\begin{gathered}
\mathrm{ai}=\mathrm{Y}_{11}-\mathrm{Y}_{10} \mathrm{Que} \text { es la diferencia entre } \\
\text { ambos resultados }
\end{gathered}
$$

Esta forma de ver el impacto del programa en el individuo " $i$ " lleva al problema fundamental de identificación, ya que es imposible que el individuo "i" presente ambos estados al mismo tiempo, debido a que solo puede tener uno de los 2 estados y no puede recibir sin haber recibido el tratamiento. Si se tiene un resultado disponible, no se puede tener el otro. Sin embargo es inferible.

Por ejemplo, se puede usar el "efecto promedio del tratamiento" más conocido como ATE por su sigla en inglés. De esta forma y fundamentándose en la ley de los grandes números, se podrá obtener un indicador del efecto del tratamiento, de esta forma: 


$$
\mathrm{ATE}=\mathrm{E}\left(\mathrm{Y}_{1}-\mathrm{Y}_{0}\right)
$$

Donde $Y_{1}$ es el resultado potencial de los individuos que participaron del programa, y $\mathrm{Y}_{0}$ es el resultado final de los individuos que no participaron del programa, luego de ser ejecutado el programa.

Un problema que podría enfrentar la evaluación es la existencia de sesgo de selección en los individuos. Como los individuos que recibirán el tratamiento comparten características (aprendices de educación media de liceos municipales) puede que eso altere los resultados ya que presentan diferencias de base con respecto a otros individuos de la población lo que los haría menos "comparables". Esto podría llevar a que posiblemente en ausencia del programa se cree una brecha grande entre diferentes sectores de la población, por lo que la diferencia de medias subestimaría el efecto que se quiere medir.

Para poder aislar los problemas ocasionados por el sesgo de selección y los efectos de los factores externos, es necesario realizar una selección aleatoria de los individuos que estarán en el grupo de control y los que formarán parte del grupo de tratamiento.

\section{Conclusiones}

Para concluir en los últimos años, la Centro de la Industria, la empresa y los Servicios (Sena Huila) en la sede Neiva $(\mathrm{H})$ y su Programa en Tecnología en Gestión Administrativa han mostrado un interés creciente por el proceso de Emprendimiento y la generación de empresarios regionales y por las novedades y retos que éste implica. Actualmente inmersa en un proceso de evaluación de la titulación de Tecnología en Gestión Administrativa y desu feria de Marketing y Ventas, realizada en el municipio de Neiva (H). Con éstas actividades se pretenden poner en marcha, entre otros, sistemas novedosos de evaluación a través de encuestas.

El emprendedor juega un papel importante en el desarrollo empresarial al crear empresas y contribuir con la configuración socioeconómica de las regiones, dotado de características, aspiraciones e ideales que lo impulsan a identificar opciones, sueños y la necesidad de ser independiente en función de generar proyectos por un bienestar familiar o individual convirtiéndose en actor principal en la economía y desarrollo productivo.

Los resultados a nivel de productos están orientados hacia el componente y ven el resultado inmediato del efecto directo del componente y acción del programa en sí por lo que responde a un horizonte de tiempo de corto plazo. Busca responder en qué magnitud las acciones realizadas generaron los productos que benefician directamente al grupo objetivo. En el caso de los programas de emprendimiento se podrían usar indicadores como: Cantidad de clases realizadas, porcentaje de asistencia y participación, cantidad de docentes capacitados, entre otras. La calidad de los productos entregados también puede medirse, y debe hacerse de manera objetiva y subjetiva. Por ejemplo la calidad de los materiales de apoyo entregados y la percepción de los beneficiarios sobre las clases y talleres que se realizan en los programas de emprendimiento.

Los resultados intermedios obedecen a cambios generados en el grupo objetivo a raíz de los productos entregados, por lo que corresponden a un horizonte de tiempo de mediano plazo. En el caso del emprendimiento, se pueden ver estos efectos midiendo la percepción sobre emprendimiento de los jóvenes beneficiados con el tratamiento, cambios en su disposición a iniciar un emprendimiento, aprendizaje técnico adquirido sobre cómo emprender, entre otros.

Los resultados finales del programa evaluado se miden a nivel de propósito o fin del programa, por lo que corresponden a un horizonte de tiempo de largo plazo. Deben mostrar un mejoramiento significativo y perdurable en el tiempo de las características sobre las cuales se quería trabajar en el diseño del programa. En el caso del emprendimiento, dependiendo del fin que tenga el programa, podría significar cambios en la cultura emprendedora, el nivel de empleo, cambios en el nivel de ingresos, disminución de la pobreza, entre otros. 
En el desarrollo de esta investigación se realizó una revisión de la literatura empírica existente en relación a este tema, en la que se aprecia la importancia de contar con una línea base que permita realizar evaluaciones de impacto en el futuro.

Como consideración futura, sería interesante realizar una evaluación de costos versus beneficios y para eso sería necesario proponer un método para valorizar los beneficios del emprendimiento, lo que supone un desafío complejo que necesitará de otra investigación con métodos acorde a la propuesta planteada.

\section{Referencias}

Berner, H. (2009). Metodología Evaluación de Impacto. Recuperado de http://www. dipres.gob.cl/594/articles-111763_doc_ pdf_metodologia.pdf

DRAE. (2001). Diccionario de la lengua española. (versión electrónica). Madrid, España: Real Academia Española, http://lema.rae. es/drae/?val=emprender.

Formichella, María Marta. “El concepto de emprendimiento y su relación con la educación, el empleo y el desarrollo local". Monografía.

Global Entrepreneurship Monitor. GEM Colombia 2010. http://www.gemcolombia. org.

Groos, Manuel. Motivación "El modelo de las tres necesidades de David McClelland". 2009. http://manuelgross.bligoo.com/content/ view/615051/Motivacion-El-Modelode-las-Tres-Necesidades-de-DavidMcClelland.html. Fecha de consulta: 1 de marzo de 2009.

Gutiérrez, Guillermo Antonio. "Habilidades del emprendedor y características emprendedoras personales". Guía fácil para emprendedores. Aulafácil.com. 2009. http://www.aulafacil.com/emprendedores-
guia-facil/curso/Lecc-6.htm. Fecha de consulta: 2 de marzo de 2009.

Instituto Nacional de Tecnología Agropecuaria, 2004. http://www.inta.gov.ar/barrow/ info/documentos/agroindustria/ MonografiaVersionFinal.pdf. Fecha de consulta: 2 de abril de 2009.

Ministerio de Comercio, Industria y Turismo. (2004). Ley 905 por medio de la cual se modifica la Ley 590 de 2000 sobre promoción del desarrollo de la micro, pequeña y mediana empresa colombiana y se dictan otras disposiciones. Recuperado de http:// www.alcaldiabogota.gov.co/sisjur/normas/ Norma1. jsp?i=14501

Ministerio de Comercio, Industria y Turismo. (2006). Ley 1014 de fomento a la cultura del emprendimiento. Recuperado de http://www.mineducacion.gov.co/1621/ articles-94653_archivo_pdf.pdf

Ministerio de Hacienda. (1996). Ley 344 por la cual se dictan normas tendientes a racionalización del gasto público, se conceden unas facultades extraordinarias y se expiden otras disposiciones. Recuperado de http://www.alcaldiabogota.gov.co/sisjur/ normas/Norma1.jsp?i=345

Ministerio de Hacienda. (2000). Ley 590 por la cual se dictan disposiciones para promover el desarrollo de las micro, pequeñas y medianas empresa. Recuperado de http://www.alcaldiabogota.gov.co/sisjur/ normas/ Norma1.jsp?i=12672

Ministerio de Protección Social. (2003). Decreto 934 por el cual se reglamenta el funcionamiento del Fondo Emprender FE. Recuperado de http://www.alcaldiabogota. gov.co/sisjur/normas/Norma1.jsp?i=7596

MundoUniversitario. Recuperadodehttp://www. saber.ula.ve/bitstream/123456789/29657/1/ articulo12.pdf 
Selamé, Teresita. Emprendimiento juvenil. Santiago: INJUV, 1999. http://www.ilo.org/ public/spanish/region/ampro/cinterfor/ temas/youth/doc/not/libro275/libro275. pdf. Fecha de consulta: 21 de abril de 2009.

Shapero, A. (1975). TheDisplaced, Uncomfortable Entrepreneur. Psychology Today, (9), 83-133.
UNESCO. (2009, 05 de Julio). II Conferencia Mundial de Educación Superior.

Varela, R. (2001). Innovación empresarial, arte y ciencia en la creación de empresas. New York. Prentice Hall. 